\title{
NUMERICAL STUDY BY A CONTROLLABILITY METHOD FOR THE CALCULATION OF THE TIME-PERIODIC SOLUTIONS OF THE MAXWELL AND VLASOV-MAXWELL SYSTEMS
}

\author{
Minai Bostan ${ }^{1}$
}

\begin{abstract}
The topic of this paper is the numerical analysis of time periodic solution for electromagnetic phenomena. The Limit Absorption Method ( $L A M)$ which forms the basis of our study is presented. Theoretical results have been proved in the linear finite dimensional case. This method is applied to scattering problems and transport of charged particles.
\end{abstract}

Mathematics Subject Classification. 34A30, 35G60, 35F30.

Received: December 16, 1999. Revised: July 24, 2000; December 1st, 2000.

\section{INTRODUCTION}

In theoretical and numerical studies of the transport of charged particles, the most frequently considered model is the Vlasov-Maxwell or Vlasov-Poisson system. The main result on this topic has been obtained by DiPerna and Lions in [15]. They prove existence of weak global solution for the Cauchy problem of the VlasovMaxwell system. The existence of classical global solution is still an open problem. By neglecting the magnetic field, a simpler model is obtained, known as the Vlasov-Poisson system. Mathematical studies on Vlasov-Poisson system have been carried out by Arsenev [1] (existence of the weak global solution for the Cauchy problem), Degond [14] and Ukai [2] (existence of classical solution in two dimensions), Pfaffelmoser in [21] (existence of classical solution in three dimensions), Lions and Perthame [19] (propagation of moments and regularity results in three dimensions). Permanent regimes are particularly important. They are modeled by stationary solutions or time periodic solutions. Results concerning stationary problems can be found in the paper of Greengard and Raviart [16] for the Vlasov-Poisson system in one dimension and in [22] for the Vlasov-Maxwell system in any space dimension. The periodic Vlasov-Poisson system has been studied in [6] for one space dimension and in [7] for any arbitrary dimension. The Vlasov-Maxwell system in one dimension has been analyzed in [8]. One strong motivation for studying such solutions is the great difficulty to compute them numerically. In order to accelerate the convergence to the permanent regimes, the concept of minimal solution is used (previously introduced in [16] and [22]). A new method based on feedback control, called the Limit Absorption Method has been developed for the computation of the periodic solutions. Controllability techniques have been also considered by other authors in order to speed up the convergence to the periodic states. A least-squares/shooting approach is presented in [9] in the general context of time periodic evolution equations. Numerical results for scattering problems can be found in the same article.

Keywords and phrases. Electro-magnetism, Maxwell equations, Vlasov equation, finite volumes.

1 INRIA, 2004 route des lucioles, BP 93, 06902 Sophia-Antipolis, France. e-mail: mbostan@amadeus.net

(C) EDP Sciences, SMAI 2001 
The paper is organized as follows. In Section 2 we analyse evolution problem with time periodic source term in the linear finite dimensional case. The limit absorption concept is introduced and the main results of convergence are presented. In the next section the Limit Absorption Method is used for numerical computation of periodic solutions for the Maxwell system. The good behaviour of this method is illustrated by numerical simulations. Finally the periodic Vlasov-Maxwell system is analyzed.

\section{TheOretical StUdy of The $L A M$}

In this section we propose to study the convergence to periodic states for solutions of evolution problems with time periodic source term. The advantages of the $L A M$ will be analyzed in term of time of convergence.

\subsection{Convergence to the periodic states}

We are concerned with the study of periodic solutions for the problem:

$$
\frac{\mathrm{d} x}{\mathrm{~d} t}+\varepsilon A x(t)=f(t), t>0
$$

with the initial condition:

$$
x(0)=x_{0},
$$

where $A$ is a linear positive definite operator (matrix) in $\mathbb{R}^{d}, f$ is a $T$ periodic function, $x_{0} \in \mathbb{R}^{d}$ is the initial data at $t=0$ and $\varepsilon>0$ is a small parameter. The analytical solution of this problem writes:

$$
x(t)=\mathrm{e}^{-\varepsilon t A} x_{0}+\int_{0}^{t} \mathrm{e}^{-\varepsilon(t-s) A} f(s) \mathrm{d} s
$$

where the exponential operator is given by the convergent sum:

$$
\mathrm{e}^{-\varepsilon t A}=I-\varepsilon t A+\frac{(\varepsilon t A)^{2}}{2 !}+\ldots+\frac{(-\varepsilon t A)^{n}}{n !}+\ldots
$$

with respect to the operator norm:

$$
\|A\|=\sup _{\|x\|=1}\|A x\|_{\mathbb{R}^{d}}
$$

In order to obtain periodic solutions, we impose $x(0)=x(T)$ which implies:

$$
\left(I-\mathrm{e}^{-\varepsilon T A}\right) x_{0}=\int_{0}^{T} \mathrm{e}^{-\varepsilon(T-s) A} f(s) \mathrm{d} s .
$$

Since $A$ is positive definite, it is easy to prove that $I-\mathrm{e}^{-\varepsilon t A}$ is invertible and therefore we obtain:

$$
x_{0}=\left(I-\mathrm{e}^{-\varepsilon T A}\right)^{-1} \int_{0}^{T} \mathrm{e}^{-\varepsilon(T-s) A} f(s) \mathrm{d} s .
$$

Therefore the periodic solution is given by the formula:

$$
x_{\infty}(t)=\mathrm{e}^{-\varepsilon t A}\left(I-\mathrm{e}^{-\varepsilon T A}\right)^{-1} \int_{0}^{T} \mathrm{e}^{-\varepsilon(T-s) A} f(s) \mathrm{d} s+\int_{0}^{t} \mathrm{e}^{-\varepsilon(t-s) A} f(s) \mathrm{d} s .
$$


Proposition 1. Let $A \in \mathcal{M}_{d}(\mathbb{R})$ be a positive definite matrix with $\alpha=\inf _{\|x\|=1}(A x, x)$. Then:

$$
\left\|\mathrm{e}^{-t A}\right\| \leq \mathrm{e}^{-t \alpha}<1
$$

Moreover $\left(I-\mathrm{e}^{-t A}\right)$ is invertible.

Proof. Let us consider the homogeneous evolution problem:

$$
\frac{\mathrm{d} x}{\mathrm{~d} t}+A x(t)=0, t>0
$$

with the initial condition:

$$
x(0)=x_{0} .
$$

The solution of this problem writes:

$$
x(t)=\mathrm{e}^{-t A} x_{0}, t>0 .
$$

Multiplying the evolution equation by $x(t)$, we get:

$$
\frac{1}{2} \frac{\mathrm{d}}{\mathrm{d} t}\|x(t)\|^{2}+(A x(t), x(t))=0
$$

Since $A$ is positive definite, we conclude that:

$$
\frac{1}{2} \frac{\mathrm{d}}{\mathrm{d} t}\|x(t)\|^{2}+\alpha\|x(t)\|^{2} \leq 0, t>0
$$

or:

$$
\frac{\mathrm{d}}{\mathrm{d} t}\|x(t)\|+\alpha\|x(t)\| \leq 0, t>0
$$

By integration we have:

$$
\|x(t)\| \leq \mathrm{e}^{-\alpha t}\left\|x_{0}\right\|
$$

which is equivalent with:

$$
\frac{\left\|\mathrm{e}^{-t A} x_{0}\right\|}{\left\|x_{0}\right\|} \leq \mathrm{e}^{-\alpha t}, x_{0} \in \mathbb{R}^{d}
$$

Therefore $\left\|\mathrm{e}^{-t A}\right\| \leq \mathrm{e}^{-\alpha t}<1, t>0$ and we can prove that:

$$
\left(I-\mathrm{e}^{-t A}\right)^{-1}=I+\mathrm{e}^{-t A}+\mathrm{e}^{-2 t A}+\ldots+\mathrm{e}^{-n t A}+\ldots
$$

Now we can estimate the difference between a solution corresponding to an arbitrary data $x_{0}$ and the periodic one:

$$
x(t)-x_{\infty}(t)=\mathrm{e}^{-\varepsilon t A}\left(x_{0}-\left(I-\mathrm{e}^{-\varepsilon T A}\right)^{-1} \int_{0}^{T} \mathrm{e}^{-\varepsilon(T-s) A} f(s) \mathrm{d} s\right), t>0 .
$$


Note that if the initial data $x_{0}$ is not exactly the value given by $(7)$, the difference between the two solutions is of order of:

$$
\left\|x(t)-x_{\infty}(t)\right\| \leq \mathrm{e}^{-\varepsilon \alpha t}\left\|x_{0}-x_{\infty 0}\right\|
$$

Hence, in order to reach a $\eta>0$ approximation of the periodic state, the computation time will be of order of:

$$
t^{\star} \approx O\left(\frac{1}{\varepsilon \alpha} \log \left(\left\|x_{0}-x_{\infty 0}\right\| / \eta\right)\right) .
$$

In particular note that for very small values of the parameter $\varepsilon$ convergence to the periodic state occurs after a very long time $t^{\star}$. In the next proposition we give a general condition which assure that the periodic solutions are bounded uniformly in respect to $\varepsilon$.

Proposition 2. Let $f \in L^{\infty}$ be a $T$ periodic function and $x_{\infty}$ be the periodic solution of the problem (1), given by (8). If $f$ satisfies:

$$
\int_{0}^{T} f(s) \mathrm{d} s=0
$$

then $x_{\infty}$ is uniformly bounded with respect to $\varepsilon$.

Proof. Since $x_{\infty}$ is periodic, it is sufficient to estimate $x_{\infty}(t)$ only for $\left.t \in\right] 0, T$. Using (8) we can write:

$$
\left\|x_{\infty}(t)\right\| \leq\left\|\left(I-\mathrm{e}^{-\varepsilon T A}\right)^{-1} \int_{0}^{T} \mathrm{e}^{-\varepsilon(T-s) A} f(s) \mathrm{d} s\right\|+T\|f\|_{L^{\infty}} .
$$

In order to finish the proof, we have to compute the limit for $\varepsilon \rightarrow 0$ :

$$
\begin{gathered}
\lim _{\varepsilon \rightarrow 0}\left(I-\mathrm{e}^{-\varepsilon T A}\right)^{-1} \int_{0}^{T} \mathrm{e}^{-\varepsilon(T-s) A} f(s) \mathrm{d} s=\lim _{\varepsilon \rightarrow 0}\left(\varepsilon T A-\frac{(\varepsilon T A)^{2}}{2 !}+\ldots\right)^{-1} \\
\int_{0}^{T}\left[I-\varepsilon(T-s) A+\frac{[\varepsilon(T-s) A]^{2}}{2 !}+\ldots\right] f(s) \mathrm{d} s=-\frac{1}{T} \int_{0}^{T}(T-s) f(s) \mathrm{d} s .
\end{gathered}
$$

Remark 1. Matrix $A$ being positive definite is also invertible.

\subsection{Limit Absorption Method}

Now consider a positive number $\lambda$ and let us introduce an absorption term in equation (1):

$$
\lambda x(t)+\frac{\mathrm{d} x}{\mathrm{~d} t}+\varepsilon A x(t)=f(t), t>0 .
$$

In this case the solution is given by:

$$
x(t)=\mathrm{e}^{-t(\varepsilon A+\lambda I)} x_{0}+\int_{0}^{t} \mathrm{e}^{-(t-s)(\varepsilon A+\lambda I)} f(s) \mathrm{d} s,
$$

and the error writes:

$$
\begin{aligned}
\left\|x(t)-x_{\infty}(t)\right\| & =\left\|\mathrm{e}^{-t(\varepsilon A+\lambda I)}\left(x_{0}-x_{\infty 0}\right)\right\| \\
& \leq \mathrm{e}^{-t(\varepsilon \alpha+\lambda)}\left\|x_{0}-x_{\infty 0}\right\|,
\end{aligned}
$$


so an $\eta$ approximation of the periodic state can be reached after a time:

$$
t^{\star} \approx O\left(\frac{1}{\varepsilon \alpha+\lambda} \log \left(\left\|x_{0}-x_{\infty 0}\right\| / \eta\right)\right)<O\left(\frac{1}{\lambda} \log \left(\left\|x_{0}-x_{\infty 0}\right\| / \eta\right)\right) .
$$

Note that in this case we have obtained an upper bound for the convergence time not depending on $\varepsilon$. We remember that the solution obtained in this case doesn't verify the initial problem (1) but a perturbation of it. The idea will be to replace the constant $\lambda>0$ by a sequence decreasing to zero. We can prove the following general result of convergence:

Proposition 3. Let $\left(\lambda_{n}\right)_{n \geq 0}$ be a decreasing sequence of positive real numbers such as:

$$
\begin{aligned}
& \text { (i) } \quad \lim _{n \rightarrow \infty} \lambda_{n}=0 \\
& \text { (ii) } \quad \Sigma_{n \geq 0} \lambda_{n}<\infty
\end{aligned}
$$

and $x_{\infty}$ be the periodic solution (8) of the problem (1). Then the evolution equation:

$$
\lambda(t) x(t)+\frac{\mathrm{d} x}{\mathrm{~d} t}+\varepsilon A x(t)=f(t), t>0,
$$

with the initial condition:

$$
x(0)=x_{0},
$$

where $f \in L^{\infty}$ is $T$ periodic and $\lambda(t)=\lambda_{n}, t \in[n T,(n+1) T[, n \geq 0$ has an unique solution which converges to the periodic state:

$$
\lim _{n \rightarrow \infty}\left\|x(n T+\cdot)-x_{\infty}(\cdot)\right\|_{L^{\infty}(0, T)}=0
$$

Proof. The solution $x(\cdot)$ is given by the formula:

$$
x(t)=\mathrm{e}^{-\int_{0}^{t}(\varepsilon A+\lambda(s) I) \mathrm{d} s} x_{0}+\int_{0}^{t} \mathrm{e}^{-\int_{s}^{t}(\varepsilon A+\lambda(\tau) I) \mathrm{d} \tau} f(s) \mathrm{d} s, t>0 .
$$

If we denote by $x_{n}$ the value of the solution at $t=n T, n \geq 0$, we can write:

$$
x(n T+t)=\mathrm{e}^{-t\left(\varepsilon A+\lambda_{n} I\right)} x(n T)+\int_{0}^{t} \mathrm{e}^{-(t-s)\left(\varepsilon A+\lambda_{n} I\right)} f(s) \mathrm{d} s, t \in[0, T[,
$$

which implies:

$$
x_{n+1}=\mathrm{e}^{-T\left(\varepsilon A+\lambda_{n} I\right)} x_{n}+\int_{0}^{T} \mathrm{e}^{-(T-s)\left(\varepsilon A+\lambda_{n} I\right)} f(s) \mathrm{d} s, n \geq 0 .
$$

Let us put $Q_{n}=\mathrm{e}^{-T\left(\varepsilon A+\lambda_{n} I\right)}$ and $B_{n}=\int_{0}^{T} \mathrm{e}^{-(T-s)\left(\varepsilon A+\lambda_{n} I\right)} f(s) \mathrm{d} s$. Obviously we have:

$$
\lim _{n \rightarrow \infty} Q_{n}=Q:=\mathrm{e}^{-T \varepsilon A},
$$

and:

$$
\lim _{n \rightarrow \infty} B_{n}=B:=\int_{0}^{T} \mathrm{e}^{-(T-s) \varepsilon A} f(s) \mathrm{d} s
$$


Using (21) we can check that $x(\cdot)$ is uniformly bounded:

$$
\begin{aligned}
\|x(t)\| & \leq\left\|x_{0}\right\|+\|f\|_{L^{\infty}} \int_{0}^{t} \mathrm{e}^{-\varepsilon \alpha(t-s)} \mathrm{d} s \\
& \leq\left\|x_{0}\right\|+\|f\|_{L^{\infty}} /(\varepsilon \alpha) .
\end{aligned}
$$

With the above notations, (23) becomes:

$$
x_{n+1}=Q_{n} x_{n}+B_{n},
$$

which also writes:

$$
\begin{aligned}
x_{n+1}= & Q_{n} Q_{n-1} \ldots Q_{0} x_{0}+Q_{n} Q_{n-1} \ldots Q_{1} B_{0}+Q_{n} Q_{n-1} \ldots Q_{2} B_{1} \\
& +Q_{n} Q_{n-1} \ldots Q_{3} B_{2}+\ldots+Q_{n} B_{n-1}+B_{n},
\end{aligned}
$$

or:

$$
\begin{aligned}
x_{n+1}= & Q_{n} Q_{n-1} \ldots Q_{0} x_{0}+Q_{n} Q_{n-1} \ldots Q_{1}\left(B_{0}-B\right)+Q_{n} Q_{n-1} \ldots Q_{2}\left(B_{1}-B\right) \\
& +\ldots+\left(B_{n}-B\right)+\left(Q_{n} Q_{n-1} \ldots Q_{1}+Q_{n} Q_{n-1} \ldots Q_{2}+\ldots+Q_{n}+I\right) B .
\end{aligned}
$$

Since $\left\|Q_{n}\right\|<\|Q\|<\mathrm{e}^{-T \varepsilon \alpha}<1$, we can prove that $\lim _{n \rightarrow \infty} Q_{n} \ldots Q_{0} x_{0}=0$. We now wish to show that the sum $\sum_{n \geq 0}\left\|B_{n}-B\right\|$ is convergent. Indeed, the general term is bounded by:

$$
\begin{aligned}
\left\|B_{n}-B\right\| & \leq \int_{0}^{T}\left\|\mathrm{e}^{-(T-s) \varepsilon A}\right\|\left(1-\mathrm{e}^{-(T-s) \lambda_{n}}\right)\|f(s)\| \mathrm{d} s \\
& \leq \lambda_{n} \frac{T}{\varepsilon \alpha}\|f\|_{L^{\infty}},
\end{aligned}
$$

and therefore:

$$
\sum_{n \geq 0}\left\|B_{n}-B\right\| \leq \frac{T}{\varepsilon \alpha}\|f\|_{L^{\infty}} \sum_{n \geq 0} \lambda_{n}<+\infty
$$

Since $\sum_{n \geq 0} Q^{n}$ is convergent, the convolution product is also convergent and so necessarily the general term converge to zero:

$$
\lim _{n \rightarrow \infty}\left\|Q^{n}\right\|\left\|B_{0}-B\right\|+\left\|Q^{n-1}\right\|\left\|B_{n-1}-B\right\|+\ldots+\left\|B_{n}-B\right\|=0
$$

and moreover:

$$
\lim _{n \rightarrow \infty} Q_{n} Q_{n-1} \ldots Q_{1}\left(B_{0}-B\right)+Q_{n} Q_{n-1} \ldots Q_{2}\left(B_{1}-B\right)+\ldots+\left(B_{n}-B\right)=0 .
$$

We prove now that the sequence $Y_{n}=I+Q_{n}+Q_{n} Q_{n-1}+\ldots+Q_{n} Q_{n-1} \ldots Q_{1}$ is convergent:

$$
Y_{n}=I+Q+\ldots Q^{n}+\left(\mathrm{e}^{-\lambda_{n} T}-1\right) Q+\ldots+\left(\mathrm{e}^{-\left(\lambda_{n}+\ldots \lambda_{1}\right) T}-1\right) Q^{n} .
$$

Let us consider $\delta>0$. Taking into account (ii) we can choose $k$ and $N$ such as:

$$
\lambda_{k+1}+\lambda_{k+2}+\ldots+\lambda_{n}<\frac{\delta}{2}, \quad n>k,
$$


and:

$$
\|Q\|^{n-k}<\frac{\delta}{2}, \quad n>N
$$

We can write:

$$
\left\|\left(\mathrm{e}^{-\lambda_{n} T}-1\right) Q+\ldots+\left(\mathrm{e}^{-\left(\lambda_{n}+\ldots \lambda_{1}\right) T}-1\right) Q^{n}\right\| \leq T\left(\lambda_{n}\|Q\|+\ldots+\left(\lambda_{n}+\ldots+\lambda_{1}\right)\left\|Q^{n}\right\|\right) .
$$

Using now (33) and (34) we obtain:

$$
\begin{aligned}
\lambda_{n}\|Q\|+\ldots+\left(\lambda_{n}+\ldots+\lambda_{1}\right)\left\|Q^{n}\right\|= & \lambda_{n}\|Q\|+\ldots+\left(\lambda_{n}+\ldots+\lambda_{k+1}\right)\|Q\|^{n-k} \\
& +\left(\lambda_{n}+\ldots+\lambda_{k}\right)\|Q\|^{n+1-k}+\ldots+\left(\lambda_{n}+\ldots+\lambda_{1}\right)\|Q\|^{n} \\
\leq & \left(\lambda_{n}+\ldots+\lambda_{k+1}\right)\left(\|Q\|+\|Q\|^{2}+\ldots+\|Q\|^{n-k}\right) \\
& +\left(\lambda_{n}+\ldots+\lambda_{1}\right)\|Q\|^{n+1-k}\left(1+\|Q\|+\ldots+\|Q\|^{k-1}\right) \\
\leq & \frac{\delta}{2} \frac{\|Q\|}{1-\|Q\|}\left(1+\sum_{n \geq 0} \lambda_{n}\right), n>N .
\end{aligned}
$$

Therefore we deduce that $\lim _{n \rightarrow \infty} Y_{n}=(I-Q)^{-1}$. Finally (28) and (31) allow us to write:

$$
\lim _{n \rightarrow \infty} x_{n}=(I-Q)^{-1} B
$$

which implies:

$$
\lim _{n \rightarrow \infty}\left(x_{n}-x_{\infty}(n T)\right)=0,
$$

because $x_{\infty}(n T)=(I-Q)^{-1} B, n \geq 0$. We can now easily finish the proof using (8) et (22):

$$
\begin{aligned}
\left\|x(n T+t)-x_{\infty}(t)\right\| \leq & \left\|x(n T)-x_{\infty}(n T)\right\|+\left(1-\mathrm{e}^{-\lambda_{n} t}\right)\left\|x_{\infty}(n T)\right\| \\
& +\int_{0}^{t}\|f(s)\| \mathrm{e}^{-\varepsilon \alpha(t-s)}\left(1-\mathrm{e}^{-\lambda_{n}(t-s)}\right) \mathrm{d} s \\
\leq & \left\|x_{n}-x_{\infty}(n T)\right\|+\lambda_{n} T\left\|x_{\infty}\right\|_{L^{\infty}}+\lambda_{n} T\|f\|_{L^{1}(0, T)} .
\end{aligned}
$$

Passing to the limit for $n \rightarrow \infty$, we obtain (20), which concludes the proof. The same result can be found in the continuous case for a general coercive operator on a pre-hilbertian space:

Proposition 4. Let $\lambda(\cdot)$ be a decreasing positive function suchs as:

$$
\begin{aligned}
\text { (i) } \quad \lim _{t \rightarrow \infty} \lambda(t) & =0, \\
\text { (ii) } \quad \int_{0}^{\infty} \lambda(t) & <\infty,
\end{aligned}
$$

and $x_{\infty}$ be the periodic solution (8) of the problem (1). Then the evolution equation:

$$
\lambda(t) x(t)+\frac{\mathrm{d} x}{\mathrm{~d} t}+\varepsilon A x(t)=f(t), t>0,
$$


with the initial condition:

$$
x(0)=x_{0},
$$

where $f \in L^{\infty}$ is $T$ periodic has an unique solution which converges to the periodic state:

$$
\lim _{n \rightarrow \infty}\left\|x(n T+\cdot)-x_{\infty}(\cdot)\right\|_{L^{\infty}(0, T)}=0
$$

Proof. Obviously we can write:

$$
\lambda(t)\left(x(t)-x_{\infty}(t)\right)+\frac{\mathrm{d}}{\mathrm{d} t}\left(x-x_{\infty}\right)+\varepsilon\left(A x-A x_{\infty}\right)=-\lambda(t) x_{\infty}(t), t>0 .
$$

Multiplying scalarly the above equation by $\left(x(t)-x_{\infty}(t)\right)$ we obtain:

$$
\lambda(t)\left\|x(t)-x_{\infty}(t)\right\|^{2}+\frac{1}{2} \frac{\mathrm{d}}{\mathrm{d} t}\left\|x-x_{\infty}\right\|^{2}+\varepsilon\left(A x-A x_{\infty}, x-x_{\infty}\right)=-\lambda\left(x_{\infty}, x-x_{\infty}\right) .
$$

Now using the coercivity of $A$ we deduce:

$$
\lambda(t)\left\|x(t)-x_{\infty}(t)\right\|+\frac{\mathrm{d}}{\mathrm{d} t}\left\|x-x_{\infty}\right\|+\varepsilon \alpha\left\|x-x_{\infty}\right\| \leq \lambda\left\|x_{\infty}\right\| .
$$

By standard computation we find:

$$
\left\|x(t)-x_{\infty}(t)\right\| \leq \mathrm{e}^{-\int_{0}^{t}(\lambda(s)+\varepsilon \alpha) \mathrm{d} s}\left\|x_{0}-x_{\infty 0}\right\|+\int_{0}^{t} \mathrm{e}^{-\int_{s}^{t}(\lambda(\tau)+\varepsilon \alpha) \mathrm{d} \tau} \lambda(s)\left\|x_{\infty}(s)\right\| \mathrm{d} s .
$$

Obviously $\lim _{t \rightarrow \infty} \mathrm{e}^{-\int_{0}^{t}(\lambda(s)+\varepsilon \alpha) \mathrm{d} s}=0$. As previously we can deduce that $x_{\infty}$ is uniformly bounded and therefore, in order to complete the proof we have to show that $\lim _{t \rightarrow \infty} I(t)=\lim _{t \rightarrow \infty} \int_{0}^{t} \mathrm{e}^{-\int_{s}^{t}(\lambda(\tau)+\varepsilon \alpha) \mathrm{d} \tau} \lambda(s) \mathrm{d} s=0$. Taking into account (ii) we can choose $t_{1}$ such as:

$$
\int_{t_{1}}^{s} \lambda(\tau) \mathrm{d} \tau \leq \eta, \quad s>t_{1} .
$$

We can write:

$$
\begin{aligned}
I(t) & =\int_{0}^{t} \mathrm{e}^{-\int_{s}^{t}(\lambda(\tau)+\varepsilon \alpha) \mathrm{d} \tau} \lambda(s) \mathrm{d} s \\
& =\int_{0}^{t_{1}} \mathrm{e}^{-\int_{s}^{t}(\lambda(\tau)+\varepsilon \alpha) \mathrm{d} \tau} \lambda(s) \mathrm{d} s+\int_{t_{1}}^{t} \mathrm{e}^{-\int_{s}^{t}(\lambda(\tau)+\varepsilon \alpha) \mathrm{d} \tau} \lambda(s) \mathrm{d} s \\
& =I_{1}(t)+I_{2}(t) .
\end{aligned}
$$

Since $\lambda(t)$ is a decreasing function we obtain:

$$
\begin{aligned}
I_{1}(t) & \leq \lambda(0) \int_{0}^{t_{1}} \mathrm{e}^{-\varepsilon \alpha(t-s)} \mathrm{d} s \\
& =\frac{\lambda(0)}{\varepsilon \alpha}\left[\mathrm{e}^{-\varepsilon \alpha\left(t-t_{1}\right)}-\mathrm{e}^{-\varepsilon \alpha t}\right] \rightarrow 0, \text { for } t \rightarrow \infty .
\end{aligned}
$$


Finally using (43) we deduce:

$$
\begin{aligned}
I_{2}(t) & =\int_{t_{1}}^{t} \mathrm{e}^{-\int_{s}^{t}(\lambda(\tau)+\varepsilon \alpha) \mathrm{d} \tau} \lambda(s) \mathrm{d} s \\
& \leq \int_{t_{1}}^{t} \lambda(s) \mathrm{d} s \leq \eta .
\end{aligned}
$$

We define now the notion of $\xi$-approximation of the periodic state:

Definition 1. We say that a sequence of functions $x_{\xi}(t)$ is a $\xi$-approximation $(\xi>0)$ of the periodic state $x_{\infty}(t)$ if there is a constant $C>0$ such as:

$$
\left\|x_{\xi}(t)-x_{\infty}(t)\right\| \leq C \xi, \forall t, \xi
$$

Proposition 5. Let $f \in L^{\infty}$ be a periodic function which verifies (13) and $x(t)$ be solution of the equation:

$$
\left.\lambda x(t)+\frac{\mathrm{d} x}{\mathrm{~d} t}+\varepsilon A x(t)=f(t), t \in\right] 0, T[,
$$

which satisfies:

$$
\|x(T)-x(0)\| \leq \eta
$$

and $x_{\infty}(t)$ be the periodic solution given by (8). Then $x$ is an approximation of $x_{\infty}$ of order $O\left(\frac{\eta}{\lambda}+\lambda\right)$. In particular, if $\lambda=\sqrt{\eta}$, the function $x$ is a $\sqrt{\eta}$-approximation of the periodic state.

Proof. Let $\delta$ represent the difference between $x$ and $x_{\infty}$. The function $\delta$ verifies:

$$
\left.\lambda \delta(t)+\frac{\mathrm{d} \delta}{\mathrm{d} t}+\varepsilon A \delta(t)=-\lambda x_{\infty}, \quad t \in\right] 0, T[,
$$

and:

$$
\|\delta(T)-\delta(0)\| \leq \eta
$$

Let $\varphi$ be the periodic solution of the problem:

$$
\left.\lambda \varphi(t)+\frac{\mathrm{d} \varphi}{\mathrm{d} t}+\varepsilon A \varphi(t)=-x_{\infty}, \quad t \in\right] 0, T[
$$

and $\mu=\delta-\lambda \varphi$. Using (1) and (13) we deduce that $\int_{0}^{T} x_{\infty}(s) \mathrm{d} s=0$. Taking into account Proposition 2 we show that there is a constant $C>0$ such as $\|\varphi(t)\| \leq C, t \in] 0, T[, \varepsilon>0$. Using (45), (46) and (47) we obtain:

$$
\left.\lambda \mu(t)+\frac{\mathrm{d} \mu}{\mathrm{d} t}+\varepsilon A \mu(t)=0, \quad t \in\right] 0, T[
$$

and:

$$
\|\mu(T)-\mu(0)\| \leq \eta
$$

Since $\mu(t)=\mu(0) \cdot \mathrm{e}^{-t(\varepsilon A+\lambda I)}$, condition (48) implies:

$$
\|\mu(0)\| \leq\left\|\left(I-\mathrm{e}^{-T(\varepsilon A+\lambda I)}\right)^{-1}\right\| \eta \approx O\left(\frac{\eta}{(\varepsilon \alpha+\lambda) T}\right) .
$$


Finally we deduce:

$$
\begin{aligned}
\|\delta(t)\| & \leq\|\mu(t)\|+\lambda\|\varphi(t)\| \\
& \leq C \frac{\eta}{(\varepsilon \alpha+\lambda) T}+C \lambda \\
& \leq C \frac{\eta}{\lambda T}+C \lambda .
\end{aligned}
$$

Now it is clear that $\left\|x(t)-x_{\infty}(t)\right\| \approx O(\sqrt{\eta})$ if $\lambda=\sqrt{\eta}$.

Remark 2. If $A$ is positive definite, then:

$$
\left\|\left(I-\mathrm{e}^{-t(\varepsilon A+\lambda I)}\right)^{-1}\right\| \leq \frac{1}{1-\mathrm{e}^{-t(\varepsilon \alpha+\lambda)}}, \quad t>0 .
$$

Proof. Using the formula:

$$
(I-P)^{-1}=I+P+P^{2}+\ldots+P^{n}+\ldots \text { if }\|P\|<1,
$$

we can write:

$$
\begin{aligned}
\left\|\left(I-\mathrm{e}^{-t(\varepsilon A+\lambda I)}\right)^{-1}\right\| & \leq 1+\left\|\mathrm{e}^{-t(\varepsilon A+\lambda I)}\right\|+\left\|\mathrm{e}^{-2 t(\varepsilon A+\lambda I)}\right\|+\ldots \\
& \leq 1+\mathrm{e}^{-t(\varepsilon \alpha+\lambda)}+\mathrm{e}^{-2 t(\varepsilon \alpha+\lambda)}+\ldots \\
& =\frac{1}{1-\mathrm{e}^{-(\varepsilon \alpha+\lambda)}}
\end{aligned}
$$

\subsection{Estimation of the convergence time}

In this section we are interested in estimating the convergence time. The following proposition is concerned with computation of this time under some particular choice of the sequence $\left(\lambda_{n}\right)_{n \geq 0}$.

Proposition 6. With the same notations as in Proposition 3, let $f \in L^{\infty}$ be a periodic function which verifies (13) and $\lambda_{n}$ a sequence given by:

$$
\begin{aligned}
\lambda_{0} & =1, \\
\lambda_{n+1} & =\lambda_{n}, \quad \text { if }\left\|x_{n+1}-x_{n}\right\|>\eta, n \geq 0, \\
\lambda_{n+1} & =\lambda_{n} \mathrm{e}^{-c T}, \quad \text { if }\left\|x_{n+1}-x_{n}\right\| \leq \eta, n \geq 0 .
\end{aligned}
$$

Then the convergence time to a $\sqrt{\eta}$-approximation of the periodic state verifies:

$$
t^{\star}=O\left(\frac{1}{\sqrt{\eta}} \log \left(\frac{1}{\eta}\right)\right) .
$$

Proof. Obviously $\left\{\lambda_{0}, \lambda_{1}, \ldots \lambda_{n}, \ldots\right\} \subset\left\{1, \mathrm{e}^{-c T}, \mathrm{e}^{-2 c T}, \ldots\right\}$. We show that the sequence $\left(\lambda_{n}\right)_{n>0}$ is convergent to zero, which means that each value $\mathrm{e}^{-n c T}$ is taken only by a finite number of terms $\lambda_{m}$. Let $m \geq 0, n \geq 0$ such as $\lambda_{m}=\mathrm{e}^{-n c T}$ and we wish to estimate the length of the constant subsequence $\lambda_{m}=\lambda_{m+1}+\ldots+\lambda_{m+p-1}>\lambda_{m+p}$, where $\lambda_{m+p}=\mathrm{e}^{-c T} \lambda_{m+p-1}=\mathrm{e}^{-(n+1) c T}$. We can write:

$$
x_{m+k}=Q_{m} x_{m+k-1}+B_{m}, k=1,2, \ldots p,
$$

which implies:

$$
x_{m+k}=\left(I-Q_{m}\right)^{-1} B_{m}+Q_{m}^{k}\left(x_{m}-\left(1-Q_{m}\right)^{-1} B_{m}\right), k=1,2, \ldots p
$$


and therefore:

$$
x_{m+k}-x_{m+k-1}=Q_{m}^{k-1}\left(Q_{m}-I\right)\left(x_{m}-\left(I-Q_{m}\right)^{-1} B_{m}\right), k=1,2, \ldots p
$$

Taking into account the choice (50), $p$ is the largest number which verifies:

$$
\begin{aligned}
\eta<\left\|x_{m+p-1}-x_{m+p-2}\right\| & =\left\|Q_{m}^{p-2}\left(Q_{m}-I\right)\left(x_{m}-\left(I-Q_{m}\right)^{-1} B_{m}\right)\right\| \\
& \leq\left\|Q_{m}^{p}\right\| \cdot \| Q_{m}^{-2}\left(Q_{m}-I\right)\left(x_{m}-\left(I-Q_{m}\right)^{-1} B_{m} \| .\right.
\end{aligned}
$$

Using Proposition 2, we deduce that $\left(x_{m}-\left(I-Q_{m}\right)^{-1} B_{m}\right)$ is bounded and therefore there is a constant $a>0$ such as:

$$
\eta<\mathrm{e}^{-p_{m}\left(\varepsilon \alpha+\lambda_{m}\right) T} a
$$

or:

$$
p_{m}<\frac{1}{T\left(\varepsilon \alpha+\lambda_{m}\right)} \log \left(\frac{a}{\eta}\right)
$$

Hence each value is repeated no more than $\left[\frac{1}{T\left(\varepsilon \alpha+\lambda_{m}\right)} \cdot \log \left(\frac{a}{\eta}\right)\right]$ times. Now it is obvious that the sequence $\left(\lambda_{m}\right)_{m \geq 0}$ is not stationary and therefore $\lim _{m \rightarrow \infty} \lambda_{m}=0$. Moreover:

$$
\begin{aligned}
\sum_{m} \lambda_{m} & \leq \sum_{n} \lambda_{m} \frac{1}{T\left(\varepsilon \alpha+\lambda_{m}\right)} \log \left(\frac{a}{\eta}\right) \\
& =\log \left(\frac{a}{\eta}\right) \sum_{n} \frac{\mathrm{e}^{-n c T}}{T\left(\varepsilon \alpha+\mathrm{e}^{-n c T}\right)} \\
& \leq \frac{1}{\varepsilon \alpha T\left(1-\mathrm{e}^{-c T}\right)} \log \left(\frac{a}{\eta}\right)<\infty
\end{aligned}
$$

Therefore the hypotheses $(i),(i i)$ are verified and using the Proposition 3 we deduce the convergence to the periodic state. Now we wish to estimate the convergence time to a $\sqrt{\eta}$ approximation. As shown in Proposition 5 this convergence is assured if the parameter $\lambda$ is of order of $O(\sqrt{\eta})$. The convergence time is bounded by:

$$
\begin{aligned}
t^{\star} & =\sum_{\lambda_{m}>\sqrt{\eta}} T p_{m}=\sum_{\lambda_{m}>\eta} T \frac{1}{T\left(\varepsilon \alpha+\lambda_{m}\right)} \log \left(\frac{a}{\eta}\right) \\
& =\sum_{\mathrm{e}^{-n c T}>\sqrt{\eta}} \mathrm{e}^{n c T} \log \left(\frac{a}{\eta}\right) \\
& =O\left(\frac{1}{\sqrt{\eta}} \log \left(\frac{a}{\eta}\right)\right) .
\end{aligned}
$$

Remark 3. The choice (50) of the sequence $\left(\lambda_{n}\right)_{n \geq 0}$ can be done by an equation of type:

$$
\frac{\mathrm{d} \lambda}{\mathrm{d} t}+c \chi_{[0, \eta]}(\mathcal{C}(x)) \lambda(t)=0, t \in[(n+1) T,(n+2) T[,
$$

where $\mathcal{C}(x(\cdot))=\left\|x_{n+1}-x_{n}\right\|$. 
More generally, as function $\mathcal{C}$ we will choose a criterion of periodicity for the solution $x(\cdot)$. The solution of problem (1) is performed using the system:

$$
\left\{\begin{array}{lll}
\lambda(t) x(t)+\frac{\mathrm{d} x}{\mathrm{~d} t}+\varepsilon A x(t) & =f(t), & t>0, \\
\frac{\mathrm{d} \lambda}{\mathrm{d} t}+\chi_{[-\eta, \eta]}(\mathcal{C}(x(\cdot))) \lambda(t) & =0, & t>0,
\end{array}\right.
$$

with the initial condition:

$$
\left\{\begin{array}{l}
x(0)=x_{0}, \\
\lambda(0)=\lambda_{0} .
\end{array}\right.
$$

This method is called the Limit Absorption Method (LAM). If the parameter $\lambda$ is not used the method will be called the Classical Method (CM).

Remark 4. If $\lambda_{0}=0$ then problem (54) reduces exactly to (1).

Proof. The proof is straightforward using (50) in the discrete case or (53) in the continuous case.

\subsection{Numerical illustration}

In this section we compare the convergence time of the (LAM) and (CM). Let us consider equation (1) in one dimension $(d=1)$ with a periodic term source $f(t)=\cos t$ and $A=I_{\mathbb{R}}$ :

$$
\frac{\mathrm{d} x}{\mathrm{~d} t}+\varepsilon x(t)=\cos t, t>0
$$

The periodic state in this case is given by:

$$
x_{\infty}(t)=(\varepsilon \cos t+\sin t) /\left(1+\varepsilon^{2}\right), t>0 .
$$

As function $\mathcal{C}$ we choose:

$$
\mathcal{C}(x(t))=|x(n T)-x((n-1) T)|, t \in[n T,(n+1) T[.
$$

We compare the numerical solutions obtained for $\varepsilon=0.01$ and $\varepsilon=0.0001$ using the two methods. In the first case $(\varepsilon=0.01)$ a good approximation of the periodic state is obtained after 70 periods using the $(\mathrm{CM})$ and after 7 periods using the (LAM). In the second case $(\varepsilon=0.0001)$, a very long time is required to approximate the periodic regime using the (CM) (about 6000 periods), whereas, using the (LAM), a good approximation is obtained only after 7 periods. Moreover we remark that the convergence time doesn't depend on the parameter $\varepsilon$ in the case of the (LAM).

\section{Application to the Maxwell equations}

\subsection{General presentation of the Maxwell equations}

We are concerned now with the numerical solution of scattering problems for harmonic planar waves by two dimensional purely reflecting non-convex obstacles. Maxwell equations in vacuum write:

$$
\left\{\begin{array}{l}
\mu_{0} \frac{\partial \vec{H}}{\partial t}+\operatorname{rot}(\vec{E})=0 \\
\varepsilon_{0} \frac{\partial \vec{E}}{\partial t}-\operatorname{rot}(\vec{H})=0
\end{array}\right.
$$



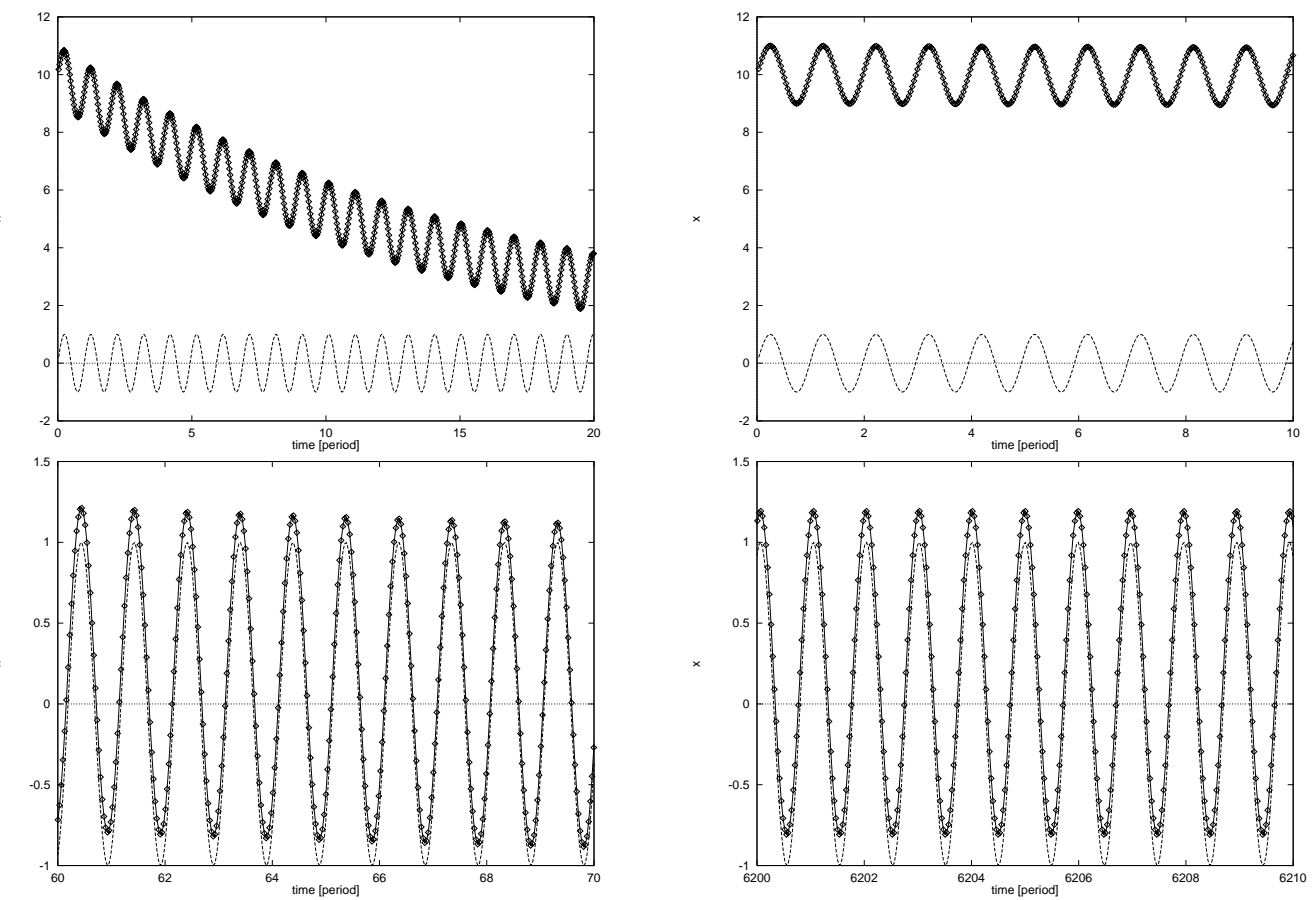

Figure 1. Convergence to the periodic state by the $C M\left(\lambda_{0}=0\right)$.
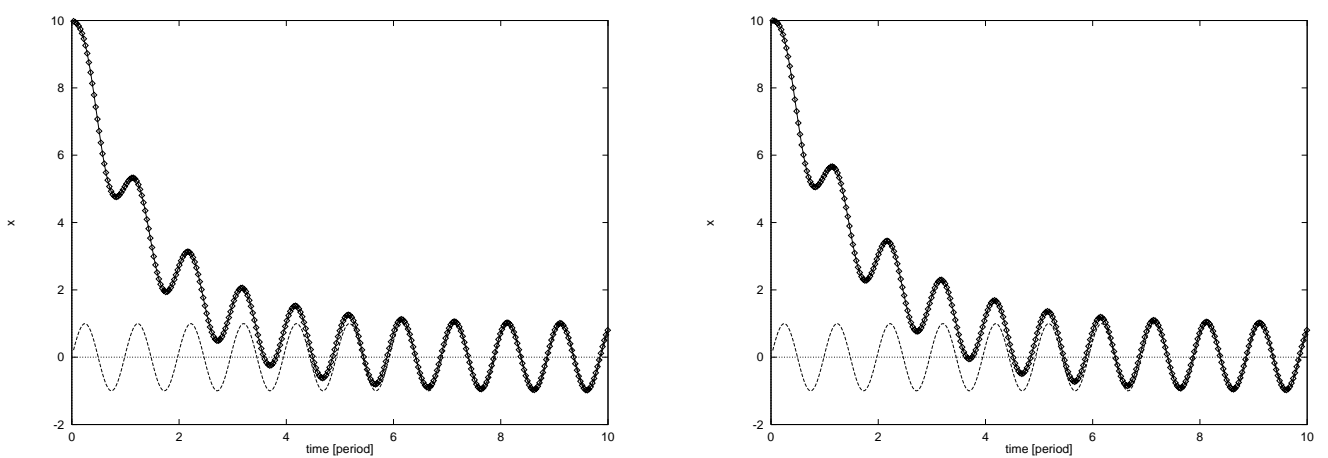

FiguRE 2. Convergence to the periodic state by the $\operatorname{LAM}\left(\lambda_{0}>0\right)$ (left: $\varepsilon=0.01 /$ right: $\varepsilon=0.0001$ ).

where $\vec{E}=\vec{E}(t, \vec{x})$ and $\vec{H}=\vec{H}(t, \vec{x})$ denote the electro-magnetic field, $\mu_{0}$ and $\varepsilon_{0}$ are respectively the permittivity and the permeability of the vacuum and $\Omega$ is the computational domain. Boundary conditions are imposed on $\Gamma=\Gamma_{\text {met }} \cup \Gamma_{\infty}$ where $\Gamma_{\text {met }}, \Gamma_{\infty}$ are respectively the metallic and artificial boundaries. Since the Maxwell system is conservative and hyperbolic, we choose the numerical approximation based on finite-volume upwind schemes [11] and [10]. In three space dimensions the conservative formulation writes:

$$
Q_{t}+\vec{\nabla} \cdot \mathbb{F}(Q)=0
$$


with:

$$
\begin{aligned}
Q & ={ }^{t}\left(H_{x}, H_{y}, H_{z}, E_{x}, E_{y}, E_{z}\right), \\
\mathbb{F}(Q) & ={ }^{t}\left(F_{1}(Q), F_{2}(Q), F_{3}(Q)\right),
\end{aligned}
$$

where:

$$
\left\{\begin{array}{l}
F_{1}(Q)={ }^{t}\left(0,-E_{z}, E_{y}, 0, H_{z},-H_{y}\right), \\
F_{2}(Q)={ }^{t}\left(E_{z}, 0,-E_{x},-H_{z}, 0, H_{x}\right), \\
F_{3}(Q)={ }^{t}\left(-E_{y}, E_{x}, 0, H_{y},-H_{x}\right) .
\end{array}\right.
$$

The weak formulation is given by:

$$
\text { Volume }\left(C_{i}\right)\left(Q_{t}\right)_{i}+\sum_{j \in K(i)} \boldsymbol{\Phi}_{\boldsymbol{i j}}+\text { boundary terms }=0
$$

where $K(i)$ denotes the set of neighbors of the node $S_{i}$ and $\operatorname{Volume}\left(C_{i}\right)$ is the volume associated to this node. The boundary terms are developed in [11]. The numerical flux is given by:

$$
\boldsymbol{\Phi}_{i j}=\boldsymbol{\Phi}\left(Q_{i}, Q_{j}, \vec{\eta}_{i j}\right)=\frac{\mathcal{F}\left(Q_{i}, \vec{\eta}_{i j}\right)+\mathcal{F}\left(Q_{j}, \vec{\eta}_{i j}\right)}{2}-\frac{\gamma}{2}\left|\mathcal{A}\left(\vec{\eta}_{i j}\right)\right|\left(Q_{j}-Q_{i}\right),
$$

where $\mathcal{F}\left(Q, \vec{\eta}_{i j}\right)=\eta_{1} F_{1}(Q)+\eta_{2} F_{2}(Q)+\eta_{3} F_{3}(Q)$ is a linear combination and $\mathcal{A}\left(\vec{\eta}_{i j}\right)$ is the Jacobian associated to $\mathcal{F}\left(Q, \vec{\eta}_{i j}\right)$. The interface values are computed using convex combination of hermitian gradients $(\beta$-scheme):

$$
\begin{aligned}
Q_{i j} & =Q_{i}+\frac{1}{2}\left\{(1-2 \beta)\left(Q_{j}-Q_{i}\right)+2 \beta \nabla Q_{i} \cdot \overrightarrow{S_{i}}\right. \\
Q_{j i} & =Q_{j}-\frac{1}{2}\left\{(1-2 \beta)\left(Q_{j}-Q_{i}\right)+2 \beta \nabla Q_{j} \cdot{\overrightarrow{S_{i}}}_{j}\right\} .
\end{aligned}
$$

The numerical experiment concerns a perfectly conducting rectangular cavity with an open crack in two space dimensions. We consider the Magnetic Transverse problem on a domain $\Omega=] 0,25 m[\times] 0,11 m[$. Absorbing conditions are imposed on $\Gamma_{\text {ext }}$ and metallic conditions on $\Gamma_{\text {int }}$. The incident field travels from right to left and is given by:

$$
\begin{aligned}
& H_{x}=-\frac{k_{y}}{|k|} \cos \left(\omega\left(t-\frac{k_{x} x+k_{y} y}{c|k|}\right)\right)-\frac{k_{y}}{|k|} \cos \left(0.5 \cdot \omega\left(t-\frac{k_{x} x+k_{y} y}{c|k|}\right)\right) \\
& H_{y}=+\frac{k_{x}}{|k|} \cos \left(\omega\left(t-\frac{k_{x} x+k_{y} y}{c|k|}\right)\right)+\frac{k_{x}}{|k|} \cos \left(0.5 \cdot \omega\left(t-\frac{k_{x} x+k_{y} y}{c|k|}\right)\right) \\
& E_{z}=\cos \left(\omega\left(t-\frac{k_{x} x+k_{y} y}{c|k|}\right)\right)+\cos \left(0.5 \cdot \omega\left(t-\frac{k_{x} x+k_{y} y}{c|k|}\right)\right)
\end{aligned}
$$

where $|k|=\omega / c=\pi \sqrt{2}, k_{x}=-|k|, k_{y}=0$. The mesh used is of the finite-element type and is non-structured (triangles). The number of points per wavelength $(=\sqrt{2})$ is approximately 15 . We show the evolution of the $H_{x}$ component of the magnetic field and of the electro-magnetic energy. In a second simulation we illuminate a perfectly conducting circular cavity by a harmonic wave. The computation domain in this case is a box 


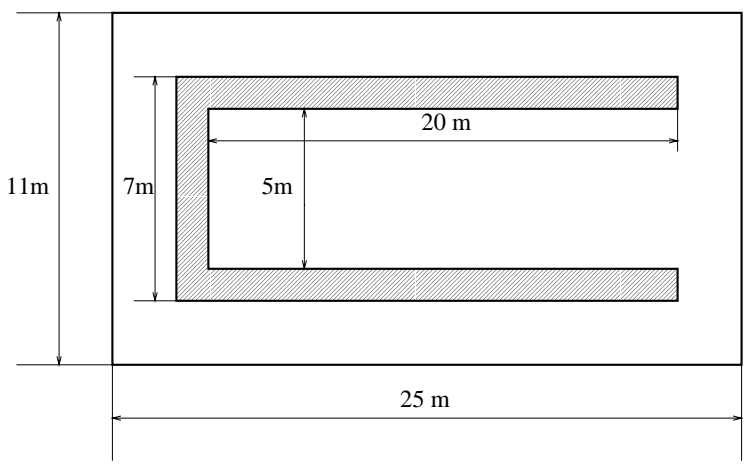

Figure 3. Rectangular cavity.

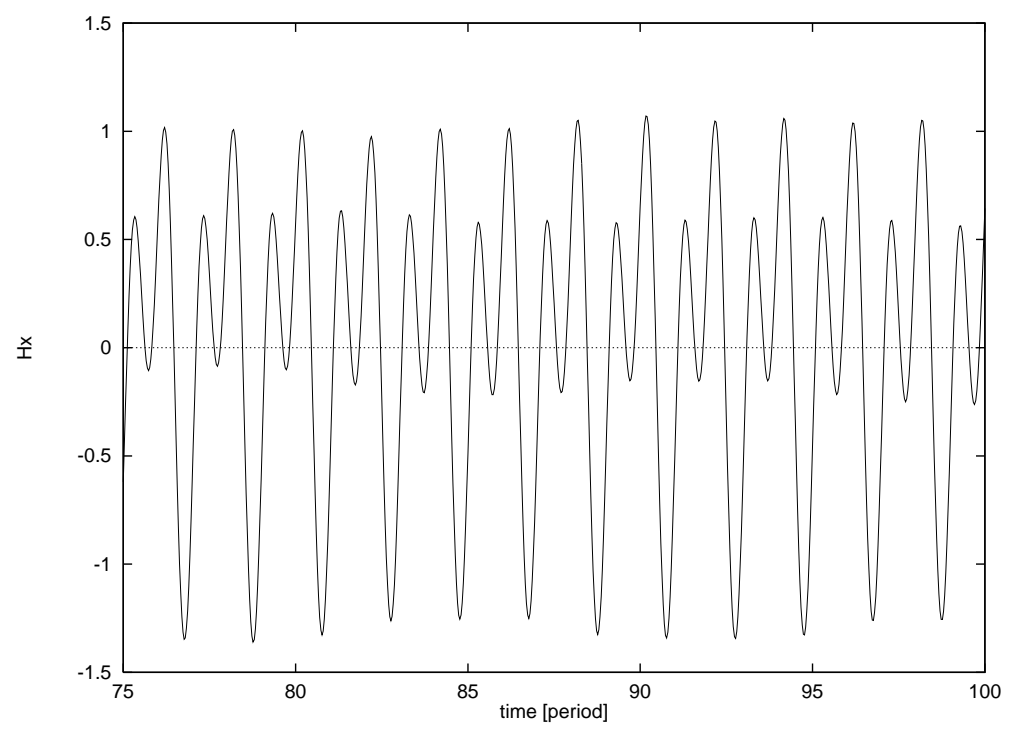

FIGURE 4. $H_{x}(t)$-rectangular cavity.

$\Omega=] 0,11 m[\times] 0,11 m[$ and the incident field is given by:

$$
\begin{aligned}
& H_{x}=-\frac{k_{y}}{|k|} \cos \left(\omega\left(t-\frac{k_{x} x+k_{y} y}{c|k|}\right)\right), \\
& H_{y}=+\frac{k_{x}}{|k|} \cos \left(\omega\left(t-\frac{k_{x} x+k_{y} y}{c|k|}\right)\right), \\
& E_{z}=\cos \left(\omega\left(t-\frac{k_{x} x+k_{y} y}{c|k|}\right)\right)
\end{aligned}
$$

with the same values for the parameters $\omega, k_{x}, k_{y}$. We observe a very poor convergence to the periodic states after 100 respectively 300 periods. In fact the convergence time of the $(C M)$ seems to be extremely large. In the following section we'll perform the same experiments by applying the $(L A M)$ in order to reduce this time of convergence. 


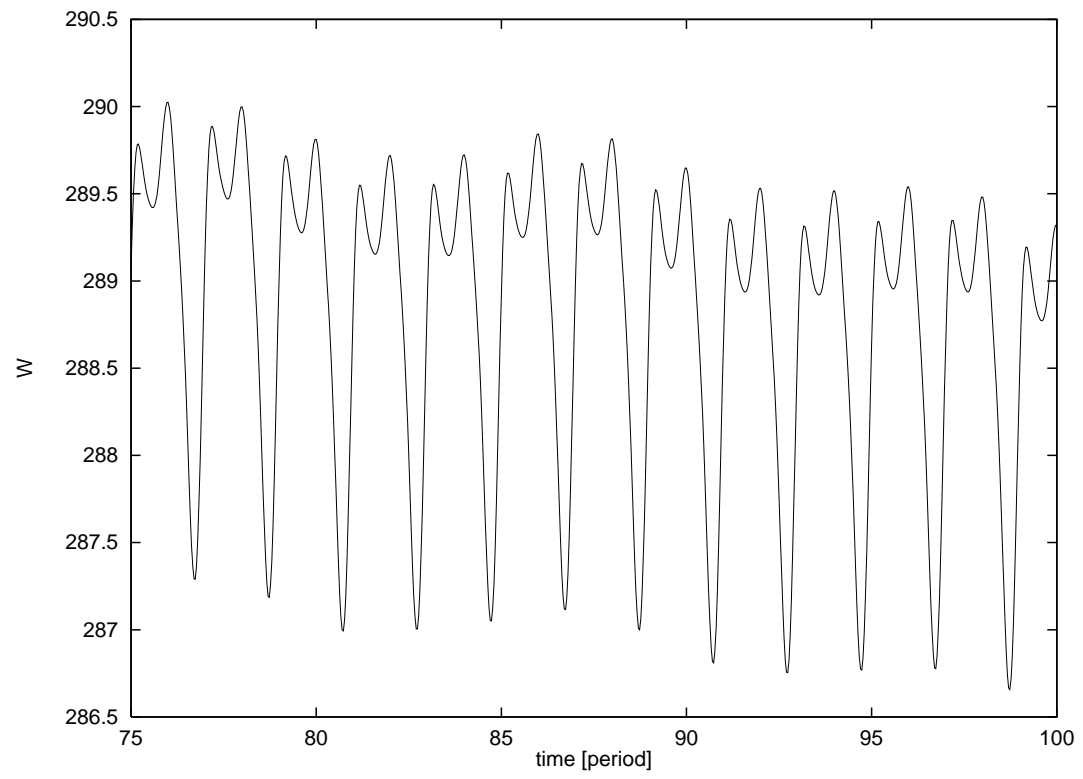

FigURE 5. Electro-magnetic energy-rectangular cavity.

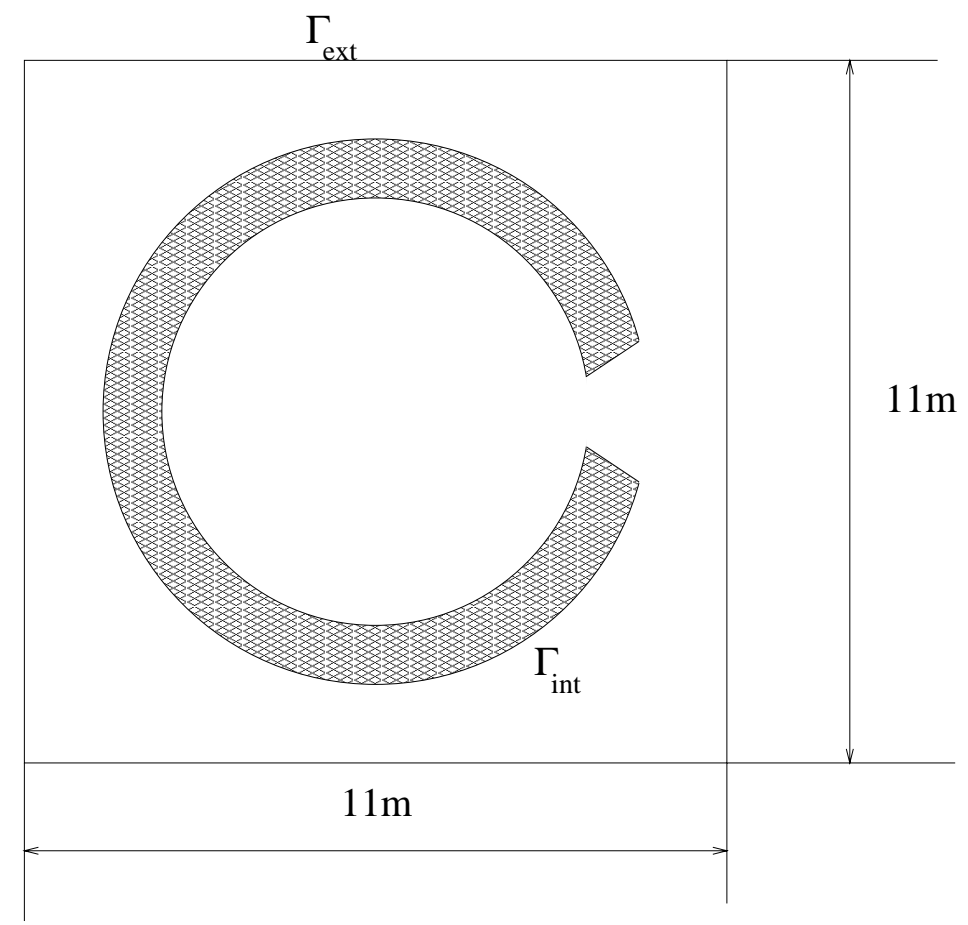

Figure 6. Circular cavity. 


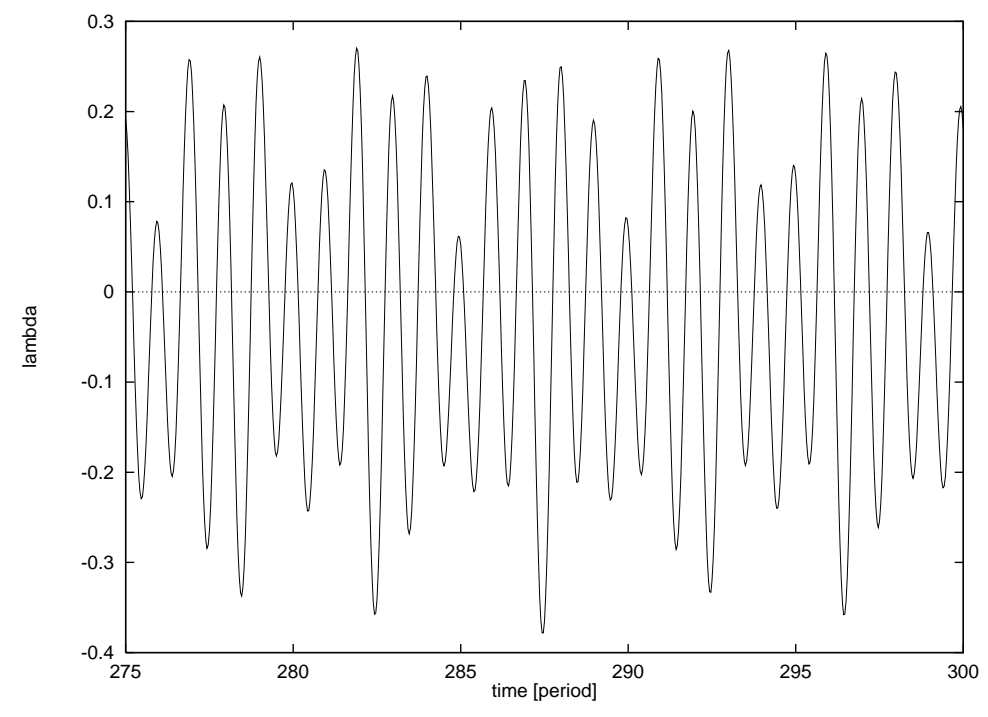

FiguRE 7. $H_{x}(t)$-circular cavity.

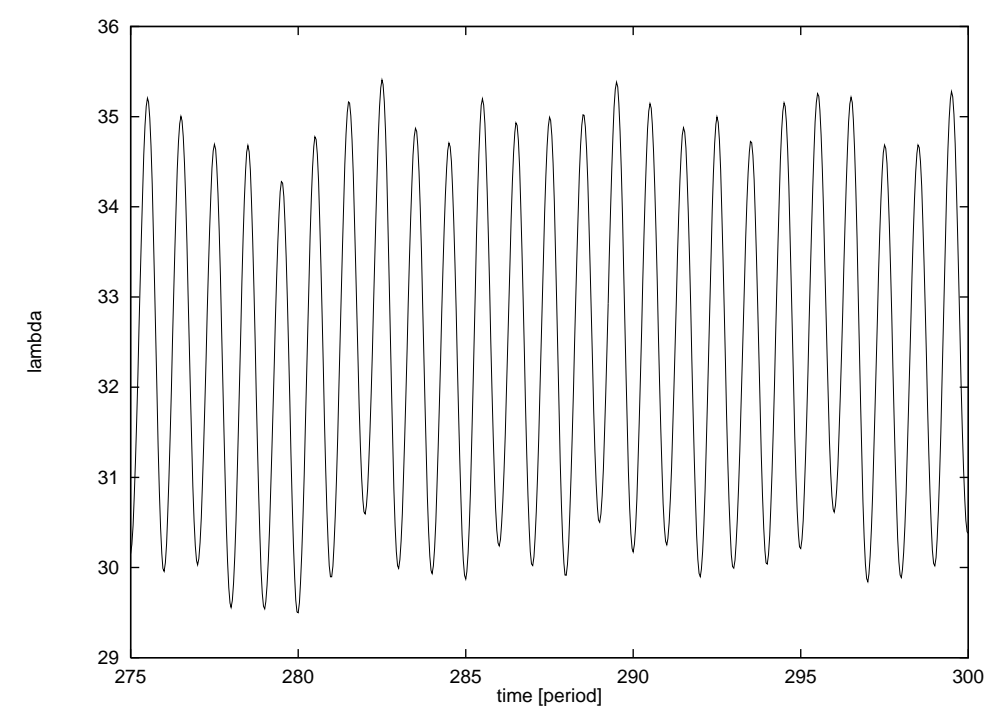

FIGURE 8. Electro-magnetic energy-circular cavity.

\section{2. $\boldsymbol{L A} \boldsymbol{M}$ for the Maxwell system}

Using the methodology previously introduced the modified Maxwell system writes:

$$
\left\{\begin{array}{l}
\lambda(t) \vec{H}+\mu_{0} \frac{\partial \vec{H}}{\partial t}+\operatorname{rot}(\vec{E})=0 \\
\lambda(t) \vec{E}+\varepsilon_{0} \frac{\partial \vec{E}}{\partial t}-\operatorname{rot}(\vec{H})=0
\end{array}\right.
$$


The time evolution of the absorption parameter $\lambda(\cdot)$ is given by an equation of type (53). When solving the periodic Maxwell system by the (LAM) two points must be considered:

$(i)$ : assure that the periodic state is well reached,

(ii): assure that the parameter $\lambda(t)$ converges very quickly to zero.

The first point $(i)$ is equivalent to the choice of a good criterion of periodicity. We'll use the criterion proposed in [20]. If the solution is harmonic:

$$
Q(t, x)=Q_{0}(x) \cos (\omega t+\varphi)
$$

then it verifies the time differential equation:

$$
\frac{\mathrm{d}^{2} Q}{\mathrm{~d} t^{2}}+\omega^{2} Q=0
$$

which implies:

$$
\int_{\Omega}\left\{\left(\frac{\mathrm{d} Q}{\mathrm{~d} t}\right)^{2}+\omega^{2} Q^{2}\right\} \mathrm{d} x=\text { const. }
$$

We propose a criterion based on the $L^{2}$ norm of the field $Q$ and its first derivative $\frac{\mathrm{d} Q}{\mathrm{~d} t}$ at each time step. The criterion $\mathcal{C}^{1}$ is given by:

$$
\mathcal{C}^{1}=\frac{\left|\int_{\Omega}\left\{\left(\frac{\mathrm{d} Q}{\mathrm{~d} t}\right)^{2}(n \mathrm{~d} t)+\omega^{2} Q^{2}(n \mathrm{~d} t)-\left(\frac{\mathrm{d} Q}{\mathrm{~d} t}\right)^{2}((n-1) \mathrm{d} t)-\omega^{2} Q^{2}((n-1) \mathrm{d} t)\right\} \mathrm{d} x\right|}{\int_{\Omega}\left\{\left(\frac{\mathrm{d} Q}{\mathrm{~d} t}\right)^{2}((n-1) \mathrm{d} t)+\omega^{2} Q^{2}((n-1) \mathrm{d} t)\right\} \mathrm{d} x} .
$$

If the field $Q$ is not harmonic, the criterion $\mathcal{C}^{1}$ will be no longer satisfied. An other criterion, more general, must be used. We can consider the energy at each period:

$$
\mathcal{C}^{2}(Q(n T))=\frac{\left|\int_{\Omega}\left\{Q^{2}(n T, x)-Q^{2}((n-1) T, x)\right\} \mathrm{d} x\right|}{\int_{\Omega} Q^{2}((n-1) T, x) \mathrm{d} x}, n \geq 1,
$$

or better:

$$
\mathcal{C}^{3}(Q(n T))=\frac{\int_{\Omega}|Q(n T, x)-Q((n-1) T, x)|^{2} \mathrm{~d} x}{\int_{\Omega} Q^{2}((n-1) T, x) \mathrm{d} x}, n \geq 1 .
$$

Now we can add to (63) the equation:

$$
\frac{\mathrm{d} \lambda}{\mathrm{d} t}+\chi_{[0, \eta]}(\mathcal{C}(H, E)) \cdot \lambda(t)=0, t>0
$$

with the initial condition:

$$
\lambda(0)=\lambda_{0}
$$

We perform the same numerical computations using now the $(L A M)$. For the rectangular cavity we choose the initial value $\lambda_{0}=0.1$ and for the circular cavity $\lambda_{0}=0.5$. As criterion we have used $\mathcal{C}^{3}$ for the combination of harmonic waves and $\mathcal{C}^{1}$ for the harmonic wave. We show the time evolution of the electro-magnetic field. We observe a good convergence to the periodic states, the value of the criterion being of order of $10^{-3}$ after 50 periods.

In the same time we have to check that the computed solutions verify also the classical Maxwell equations, which means to check that the parameter $\lambda(t)$ has a quickly decreasing to 0 . In fact, after 150 periods $\lambda(t)$ becomes of order of $10^{-5}$. 

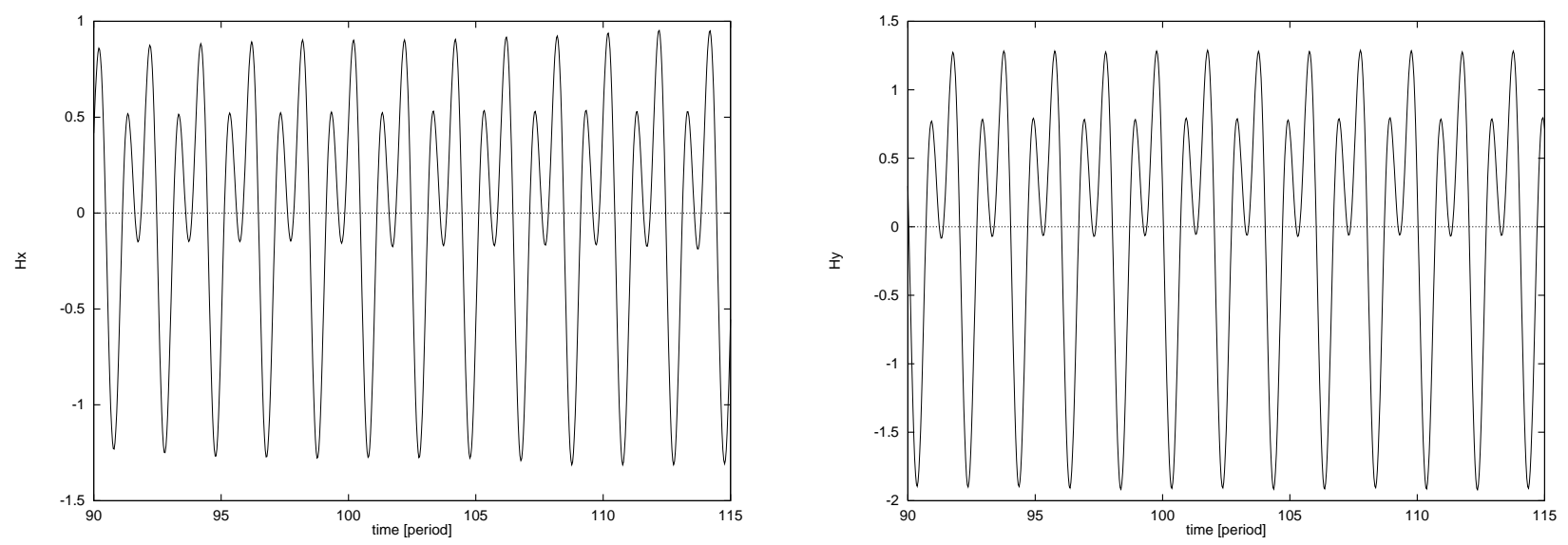

FiguRE $9 . H_{x} / H_{y}$ for the rectangular cavity.
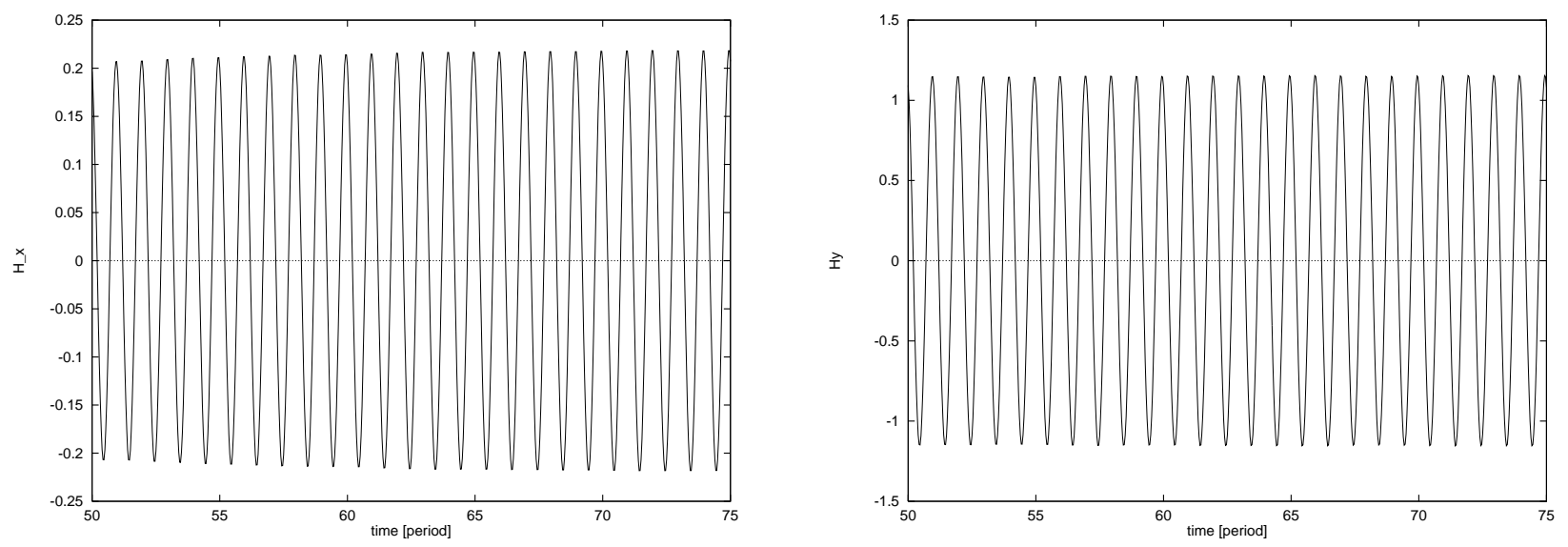

FiguRE 10. $H_{x} / H_{y}$ for the circular cavity.
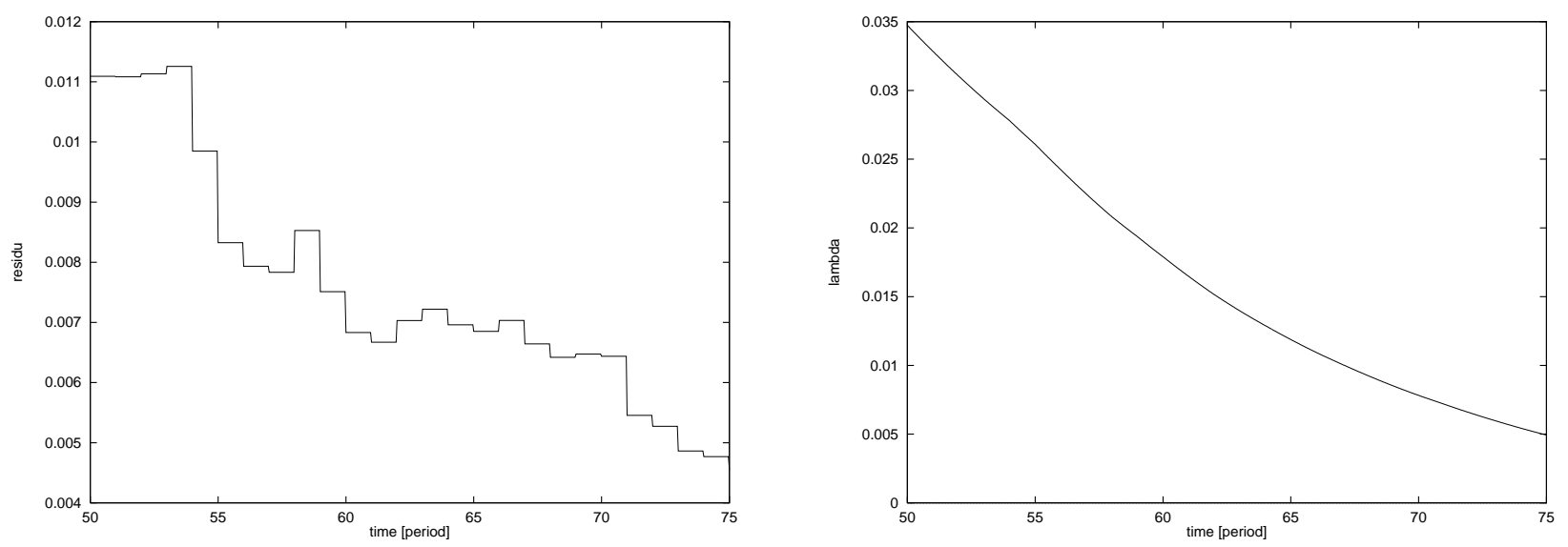

FiguRE $11 . \mathcal{C}^{1} / \lambda$ for the circular cavity. 


\section{Application of the LAM of the Vlasov-Maxwell system}

In this section we are interested by the computation of the periodic solutions for transport of charged particles in electro-magnetic field. Numerical experiences will be done using the $(C M)$ and the $(L A M)$. The convergence time of the two methods will be compared.

\subsection{General presentation of the equations}

The motion of a fluid of charged particles in an electro-magnetic field can be described in term of particle distribution function by the Vlasov equation:

$$
\partial_{t} f+v \cdot \nabla_{x} f+\frac{q}{m}\left(E+v \wedge\left(\mu_{0} H\right)\right) \cdot \nabla_{v} f=0 .
$$

The electro-magnetic field verifies the Maxwell equations:

$$
\left\{\begin{array}{c}
\mu_{0} \frac{\partial \vec{H}}{\partial t}+\operatorname{rot}(\vec{E})=0 \\
\varepsilon_{0} \frac{\partial \vec{E}}{\partial t}-\operatorname{rot}(\vec{H})=-\vec{j}
\end{array}\right.
$$

where the current density is given by:

$$
\vec{j}(t, x)=\int_{v} \vec{v} f(t, x, v) \mathrm{d} v
$$

Here we denote by $(t, x, v)$ the time, the position and the velocity, $f$ is the distribution function and $(E, H)$ is the electro-magnetic field.

The finite-volume scheme previously introduced in Section 3.1 is used for the numerical approximation of the Maxwell equations. The Vlasov equation (65) is numerically solved by means of a deterministic method. The distribution function $f$ is approximated by a combination of Dirac distributions:

$$
f(t, x, v)=\sum_{k=1}^{N} w_{k} f_{k} \delta\left(x-x_{k}(t)\right) \otimes \delta\left(v-v_{k}(t)\right)
$$

where $N$ is the number of the considered particles, $w_{k}$ is the volume associated in the phase space to the particle $k$ and $f_{k}$ is the weight of the particle $k$. The characteristic system describes the evolution of the position and the velocity $\left(x_{k}(t), v_{k}(t)\right)$ of each particle:

$$
\left\{\begin{array}{l}
\frac{\mathrm{d} x_{k}}{\mathrm{~d} t}=v_{k}(t) \\
\frac{\mathrm{d} v_{k}}{\mathrm{~d} t}=\frac{q}{m}\left(E\left(t, x_{k}(t)\right)+v_{k}(t) \wedge\left(\mu_{0} H\left(t, x_{k}(t)\right)\right)\right) .
\end{array}\right.
$$

By the definition (67) and using formula (68) we get:

$$
\vec{j}(t, x)=q \sum_{k=1}^{N} w_{k} f_{k} \vec{v}_{k}(t) \delta\left(x-x_{k}(t)\right) .
$$


Here the electro-magnetic field in each point $x_{k}(t)$ is computed using $P^{1}$ approximations and the current density in a point $S_{i}$ of the mesh is given by:

$$
\begin{aligned}
\vec{j}\left(t, S_{i}\right) & =\frac{1}{V_{i}} \int \vec{j}(t, x) \phi_{i}(x) \mathrm{d} x \\
& =\frac{q}{V_{i}} \sum_{k=1}^{N} w_{k} f_{k} \vec{v}_{k}(t) \phi_{i}\left(x_{k}(t)\right),
\end{aligned}
$$

where $V_{i}=\int \phi_{i}(x) \mathrm{d} x$ and $\phi_{i}$ is the $P^{1}$ basis function associated to the node $S_{i}$.

\subsection{Numerical results}

In this paragraph we present the numerical solutions obtained by using the $(C M)(\lambda=0)$ and the $(L A M)$ $(\lambda>0)$. The domain of computation is a rectangular box $] 0,1 \mathrm{~m}[\times] 0,1 \mathrm{~m}[$ and we consider the Electric Transverse $\left(E_{z}=0\right)$ mode. Absorbing and metallic boundary conditions are imposed as indicated in the figure. Two kinds of charged particles are injected by the left side of the box at the resonant frequency:

$$
\omega=2 \pi \nu=\frac{\pi \sqrt{2}}{\sqrt{\varepsilon_{0} \mu_{0}}} .
$$

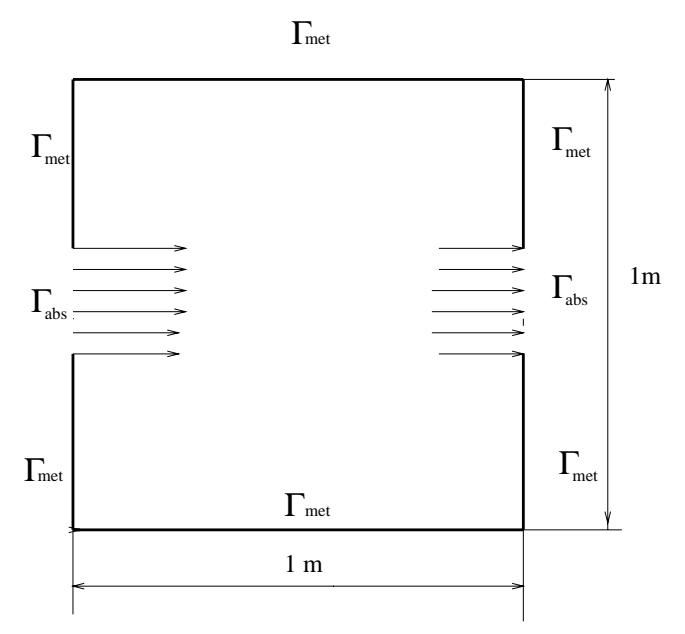

Figure 12. Metallic/Absorbing box.

The distribution functions on the boundary are given by:

$$
g_{e}(t, x, v)= \begin{cases}-|e|(2+\cos (\omega t)) \cdot \delta(v-3), & x=0, v_{x}>0 \\ 0, & \text { otherwise }\end{cases}
$$

and:

$$
g_{i}(t, x, v)= \begin{cases}+|e|(2+\sin (\omega t)) \cdot \delta(v-3), & x=0, v_{x}>0 \\ 0, & \text { otherwise }\end{cases}
$$




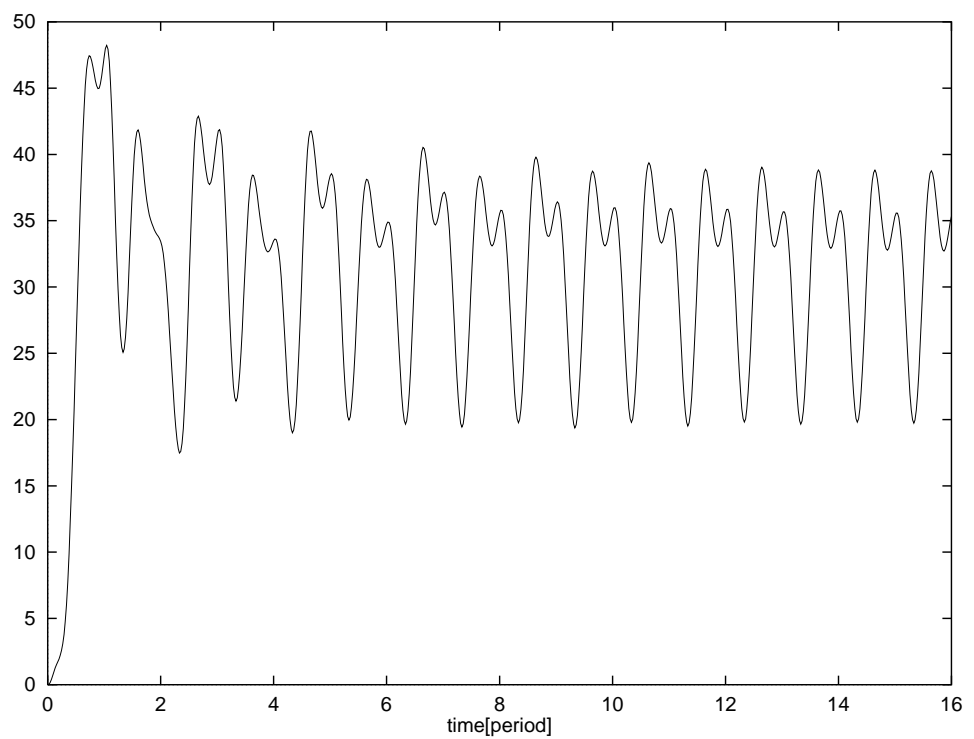

FiguRE 13. Electro-magnetic energy, $\lambda_{0}=0, \Gamma_{a b s}=50 \%$.

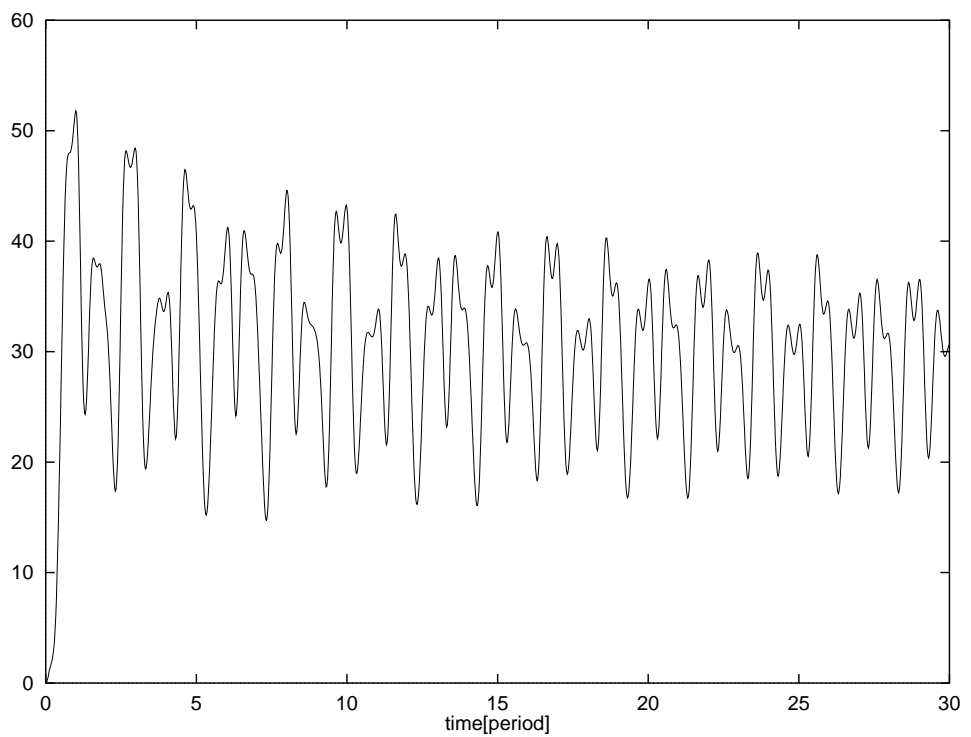

Figure 14. Electro-magnetic energy, $\lambda_{0}=0, \Gamma_{a b s}=20 \%$.

Several experiences have been done for different sizes of the injection band which represents the small parameter $\varepsilon$ introduced in Section 2.1 (the absorbing boundary conditions dissipate the energy). Using the $(C M)(\lambda=0)$ we observe a good behavior after 10 periods if $\Gamma_{a b s}$ is about $50 \%$ of the total boundary. If $\Gamma_{a b s}$ is reduced to $20 \%$ or to $10 \%$ it becomes very difficult to compute good approximations of the periodic states, the (CM) seems to be not well suited to this kind of computation. If the ( $L A M)$ is used, very good solutions are obtained in all cases which confirms the independence of the convergence time as shown in Proposition 6. Moreover, small values $\left(10^{-4}\right)$ for the parameters $\lambda(t)$ can be observed after 20 periods which assure that the solutions approximate very well the classical Vlasov-Maxwell system. 


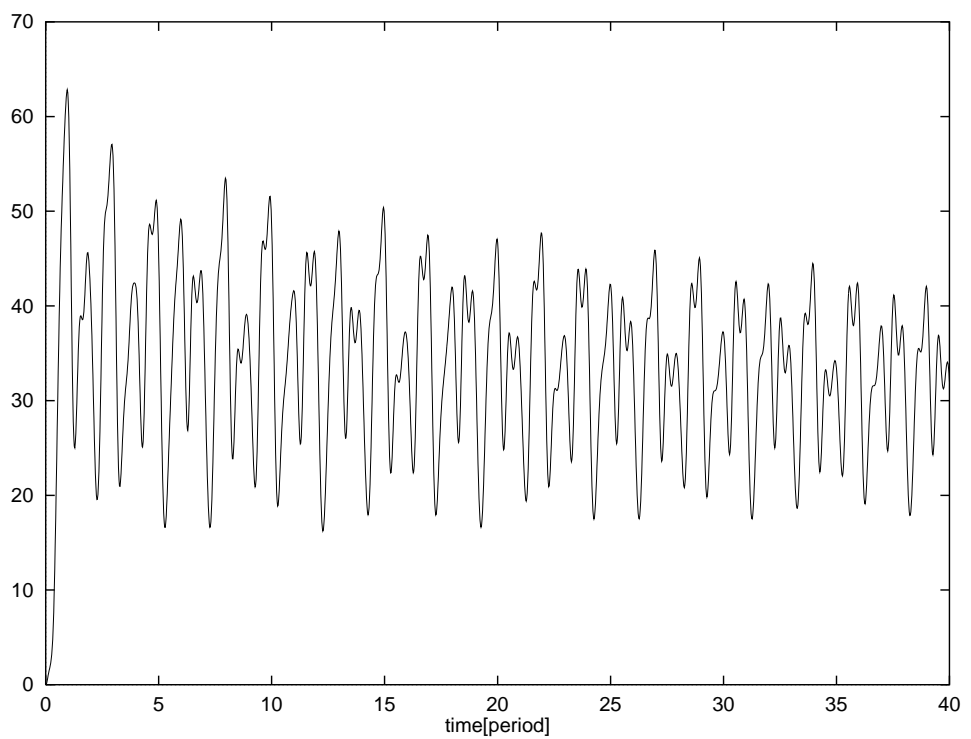

Figure 15. Electro-magnetic energy, $\lambda_{0}=0, \Gamma_{a b s}=10 \%$.

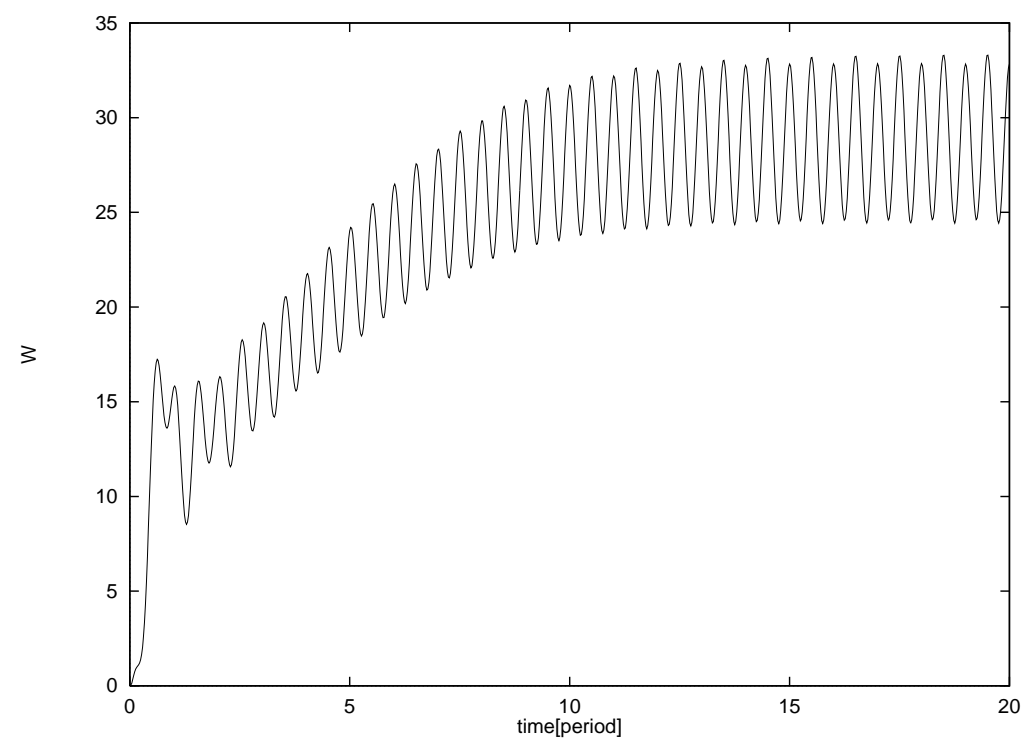

Figure 16. Electro-magnetic energy, $\lambda_{0}=0.1, \Gamma_{a b s}=10 \%$.

\section{Conclusions}

The main goal of this study was to obtain an adequate method for the computation of periodic solutions for the Maxwell and Vlasov-Maxwell systems. In order to speed-up the convergence an absorption term proportional to a control parameter $\lambda(t)$ was introduced as a perturbation of the classical equations. The time evolution of the new unknown $\lambda(t)$ is given by a control equation, using a criterion of periodicity. The main features of the new method called the Limit Absorption Method have been carried out in the linear finite dimensional case, 


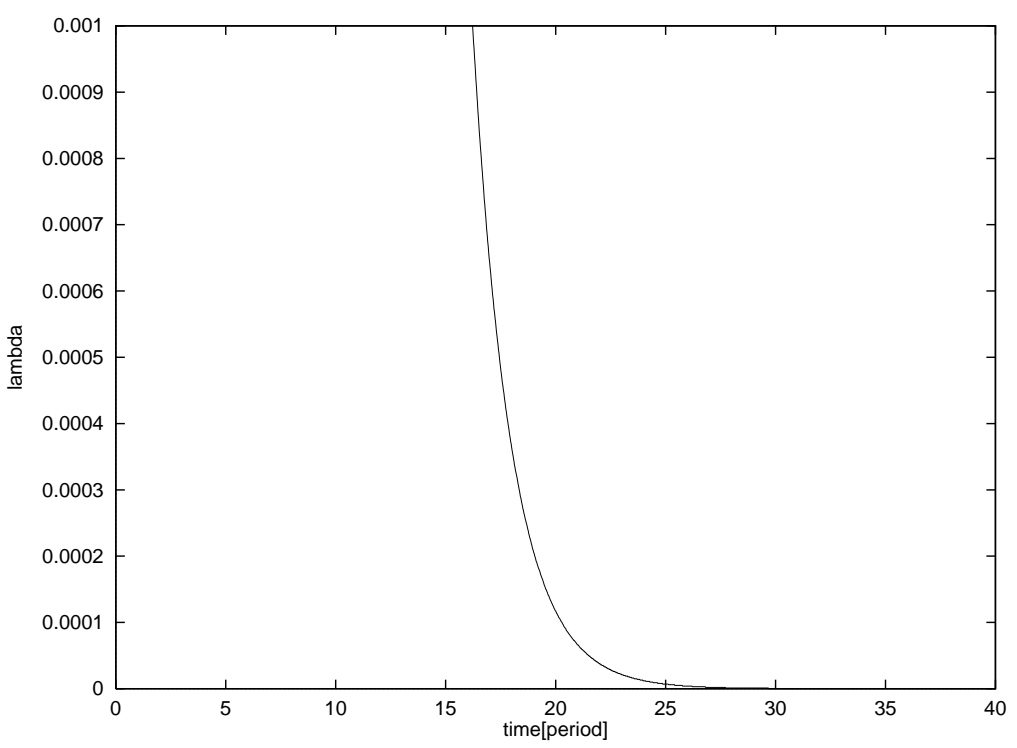

Figure 17. $\lambda=\lambda(t), \lambda_{0}=0.1, \Gamma_{a b s}=10 \%$.

as well as an estimation of the convergence time for a particular choice of the parameter $\lambda(t)$. This methodology was applied to the resolution of scattering problems for electro-magnetic waves and to the transport of charged particles in electro-magnetic field.

\section{REFERENCES}

[1] A. Arsenev, Global existence of a weak solution of Vlasov's system of equations. USSR Comp. Math. Math. Phys. 15 (1975) $131-143$.

[2] K. Asano and S. Ukai, On the Vlasov-Poisson limit of the Vlasov-Maxwell equation. Pattern and waves. Qualitative analysis of nonlinear differential equations. Stud. Math. Appl. 18 (1986) 369-383.

[3] N. Ben.Abdallah, Weak solutions of the initial-boundary value problem for the Vlasov-Poisson system,. Math. Meth. Appl. Sci. 17 (1994) 451-476.

[4] M. Bezard, Boundary value problems for the Vlasov-Maxwell system, in Semin. Équ. Deriv. Partielles, Ec. Polytech., Cent. Math., Palaiseau Semi 1992-1993, Exp. No. 4 (1993) 17.

[5] B. Bodin, Modélisation et simulation numérique du régime de Child-Langmuir. Thèse de l'École Polytechnique, Palaiseau (1995).

[6] M. Bostan and F. Poupaud, Periodic solutions of the Vlasov-Poisson system with boundary conditions. C. R. Acad. Sci. Paris, Sér. I 325 (1997) 1333-1336.

[7] M. Bostan and F. Poupaud, Periodic solutions of the Vlasov-Poisson system with boundary conditions. Math. Mod. Meth. Appl. Sci. 10 (1998) 651-672.

[8] M. Bostan and F. Poupaud, Periodic solutions of the 1D Vlasov-Maxwell system with boundary conditions. Math. Meth. Appl. Sci. 23 (2000) 1195-1221.

[9] M.O. Bristeau, R. Glowinski and J. Périaux, Controllability methods for the computation of time periodic solutions; application to scattering. J. Comp. Phys. 147 (1998) 265-292.

[10] J.P. Cioni, Résolution numérique des équations de Maxwell instationnaires par une méthode de volumes finis. Ph.D., Université de Nice Sophia-Antipolis (1995).

[11] J.P. Cioni, L. Fezoui and D. Issautier, High-order upwind schemes for solving time-domain Maxwell equation. La Recherche Aérospatiale No. 5 (1994) 319-328.

[12] P. Degond, Regularité de la solution des équations cinétiques en physiques de plasmas, in Semin. Équ. Dériv. Partielles 1985-1986, Exp. No. 18 (1986) 11.

[13] P. Degond, Local existence of solutions of the Vlasov-Maxwell equations and convergence to the Vlasov-Poisson equations for infinite light velocity. Math. Methods Appl. Sci. 8 (1986) 533-558. 
[14] P. Degond, Global existence of smooth solutions for the Vlasov-Fokker-Planck equation in 1 and 2 space dimensions. Ann. Sci. Ec. Norm. Super. IV. Ser. 19 (1986) 519-542.

[15] R.J. Diperna and P.L. Lions, Global weak solutions of Vlasov-Maxwell system. Comm. Pure Appl. Math. XVII (1989) $729-757$.

[16] C. Greengard and P.A. Raviart, A boundary value problem for the stationary Vlasov-Poisson system. Comm. Pure Appl. Math. XLIII (1990) 473-507.

[17] Y. Guo, Global weak solutions of the Vlasov-Maxwell system with boundary conditions. Comm. Math. Phys. 154 (1993) $245-263$.

[18] Y. Guo, Regularity for the Vlasov equation in a half space. Indiana Univ. Math. J. 43 (1994) 255-320.

[19] P.L. Lions and B. Perthame, Propagation of moments and regularity for the 3-dimensional Vlasov-Poisson system. Invent. Math. 105 (1991) 415-430.

[20] R. Löhner and J. Ambrosiano, A finite element solver for the Maxwell equations, in GAMNI-SMAI conference on numerical methods for the solution of Maxwell equations, Paris (1989).

[21] K. Pfaffelmoser, Global classical solutions of the Vlasov-Poisson system in 3 dimensions for general initial data. J. Diff. Eq. 95 (1992) 281-303.

[22] F. Poupaud, Boundary value problems for the stationary Vlasov-Maxwell system. Forum Math. 4 (1992) 499-527.

To access this journal online: www.edpsciences.org 